\title{
IMbrave 050: a Phase III trial of atezolizumab plus bevacizumab in high-risk hepatocellular carcinoma after curative resection or ablation
}

\author{
Stephen P Hack*,1(i), Jessica Spahn ${ }^{1}$, Minshan Chen², Ann-Lii Cheng ${ }^{3}$, Ahmed Kaseb ${ }^{4}$, \\ Masatoshi Kudo ${ }^{5}$, Han Chu Lee ${ }^{6}$, Adam Yopp ${ }^{7}$, Pierce Chow ${ }^{8}$ \& Shukui Qin ${ }^{9}$ \\ ${ }^{1}$ Genentech, Inc, 1 DNA Way, South San Francisco, CA 94080, USA \\ ${ }^{2}$ Department of Hepatobiliary Surgery, Cancer Centre of Sun Yat-sen University, Guangzhou, PR China \\ ${ }^{3}$ National Taiwan University Cancer Center \& National Taiwan University Hospital, Taipei, Taiwan \\ ${ }^{4}$ Department of Gastrointestinal Medical Oncology, The University of Texas MD Anderson Cancer Center, Houston, TX 77030, USA \\ ${ }^{5}$ Department of Gastroenterology \& Hepatology, Kindai University School of Medicine, Osaka, Japan \\ ${ }^{6}$ Department of Internal Medicine, Asan Medical Center, University of Ulsan College of Medicine, Seoul, Republic of Korea \\ ${ }^{7}$ Department of Surgery, Division of Surgical Oncology, University of Texas Southwestern Medical Center, Dallas, TX 75390, USA \\ ${ }^{8}$ Division of Surgical Oncology, National Cancer Centre, Singapore \\ ${ }^{9}$ PLA Cancer Center, People's Liberation Army (PLA) 81 Hospital, Nanjing 210016, PR China \\ *Author for correspondence: Tel.: +1 650580 7063; Fax: 650467 2327; hack.steve@gene.com
}

Hepatocellular carcinoma recurs in $70-80 \%$ of cases following potentially curative resection or ablation and the immune component of the liver microenvironment plays a key role in recurrence. Many immunosuppressive mechanisms implicated in HCC recurrence are modulated by VEGF and/or immune checkpoints such as PD-L1. Atezolizumab (PD-L1 inhibitor) plus bevacizumab (VEGF inhibitor) has been shown to significantly improve overall survival, progression-free survival and overall response rate in unresectable HCC. Dual PD-L1/VEGF blockade may be effective in reducing HCC recurrence by creating a more immunefavorable microenvironment. We describe the rationale and design of IMbrave 050 (NCT04102098), a randomized, open-label, Phase III study comparing atezolizumab plus bevacizumab versus active surveillance in HCC patients at high-risk of recurrence following curative resection or ablation. The primary end point is recurrence-free survival. Clinical Trial Registration: NCT04102098

First draft submitted: 25 February 2020; Accepted for publication: 19 March 2020; Published online: 30 April 2020

Keywords: ablation $\bullet$ adjuvant treatment $\bullet$ atezolizumab • bevacizumab • hepatocellular carcinoma $\bullet$ PD-L1 • recurrence-free survival $\bullet$ resection $\bullet$ VEGF

Hepatocellular carcinoma (HCC) accounts for approximately $90 \%$ of primary liver cancers. Globally, HCC is the third leading cause of cancer deaths, although it ranks sixth in the worldwide incidence of cancer, attesting to the poor general prognosis $[1,2]$. The pathogenesis of HCC is closely related to the presence of underlying chronic liver disease, mostly due to $\mathrm{HBV}$ or $\mathrm{HCV}$ or nonviral causes such as alcohol abuse or nonalcoholic fatty liver disease, resulting in heterogenous worldwide incidence rates. The vast majority of HCC cases $(\sim 80 \%)$ occurs in subSaharan Africa or East Asia, with more than $50 \%$ occurring in China, where the main risk factor is HBV exposure. In the USA, Europe and Japan, HCV is the main etiologic risk factor together with excessive alcohol intake. The epidemiology, pathogenesis and clinical management of HCC have been recently reviewed elsewhere $[1,2]$.

Treatment of HCC is challenging due to the presence of underlying liver dysfunction and a concomitant malignancy. In contrast to most cancers, in which treatment decisions are based primarily on tumor burden, therapeutic algorithms for HCC must account for the degree of underlying liver dysfunction and patient performance status [3]. Multiple staging criteria exist for HCC, all of which have limitations with no international consensus [4]. Barcelona Clinic Liver Cancer (BCLC) and Hong Kong Liver Cancer systems include all three of these clinical factors and include stage-dependent treatment recommendations $[5,6]$.

Future Medicine 


\section{Curative treatment options for HCC}

Curative treatments for HCC are defined as therapeutic modalities that confer a greater than $50 \%$ overall survival (OS) at 5 years and this is currently only achievable by surgical resection, orthotopic liver transplant and local ablative therapies and in relatively early HCC tumor stages.

Surgical resection represents the mainstay of curative treatment for patients with HCC who have good functional liver reserves. The criteria for selection of patients for liver resection in HCC remains hotly debated and there is currently no international consensus regarding the tumor burden and hepatic reserve that define suitability for resection. The BCLC criteria, endorsed in many Western guidelines, restrict resection to patients with very early or early stages of HCC (BCLC 0 or A) [2] and potentially deprives many patients who would benefit from curative surgery. In contrast, many Asian treatment guidelines recommend liver resection for higher burden HCC including cases with large or multifocal HCC, vascular invasion or with less optimal synthetic liver function [7]. For example, the Hong Kong criteria recommend curative resection for both early tumors $(\leq 5 \mathrm{~cm}, \leq 3$ tumor nodules with no intrahepatic venous invasion) and intermediate tumors $(\leq 5 \mathrm{~cm}$ with either $>3$ tumor nodules or intrahepatic venous invasion; or $>5 \mathrm{~cm}, \leq 3$ nodules and no vascular invasion) [5]. Controversy exists regarding which patients benefit from a surgical resection as first-line therapy. Several large retrospective series from tertiary centers have shown that up to $50 \%$ of hepatectomies are performed in patients with either intermediate (BCLC-B) or advanced stage HCC (BCLC-C) whose disease often involves multiple tumors or vascular invasion [8,9]; such patients are not considered suitable for resection according to EASL/AASLD guidelines [10,11]. Studies also indicate that outcomes are improved for selected patients with more advanced HCC when treated with resection compared with alternative treatments such as TACE $[12,13]$.

Local ablation is typically considered standard of care for patients with very early- or early-stage HCC who are not candidates for resection $[7,10,11]$. Ablation represents an alternative to surgery for single tumors between 2 and $3 \mathrm{~cm}$. The primary method of ablation is percutaneous radiofrequency ablation (RFA), which causes coagulative tumor necrosis by inducing high intratumoral temperatures $\left(60-100^{\circ} \mathrm{C}\right)$. Other ablative techniques include microwave ablation, cryoablation and ethanol injection. The extent of tumor necrosis that can be achieved by ablation is inversely correlated with tumor size and markedly reduces in tumors larger than $3 \mathrm{~cm} \mathrm{[1].}$

\section{HCC recurrence and the need for effective adjuvant treatments}

While curative for some patients, resection and ablation are associated with high rates of recurrence. Among the commonly occurring cancers, HCC is currently the only cancer with no proven adjuvant therapy after potentially curative resection. Both OS as well as disease-free survival (DFS) are thus poorer after surgical resection in early stage HCC compared with resection for early stage cancer in other common cancers such as breast or colorectal cancer. According to a recent systematic review, median 5-year DFS rates following resection in patients with early-stage HCC defined by the Milan Criteria, who are considered optimal candidates for surgery, was reported to be 37\% [14]. In resected patients with more advanced HCC for example those with large/multinodular presentation or with portal vein invasion, published 5-year DFS rates are less than 30\% [9,15]. RFA is also marred by 5 -year recurrence of up to $80 \%$ [16]. Surgical resection provides better clinical outcome than local ablation particularly among patients with well-preserved hepatic function [16].

HCC recurrence following resection is generally classified as 'early' or 'late' recurrence using the 2 years post resection time point as a cut off [17]. Early recurrence (within 2 years of resection) account for more than $70 \%$ of tumor recurrences and is generally believed to represent pre-existing intrahepatic metastasis disseminated from the resected primary tumor, whereas late recurrence ( 2 or more years following surgery) is often regarded as de novo tumor [10]. Occult intrahepatic metastasis have been observed in $37-42 \%$ of explant specimens [18]. Different risk factors are thought to contribute to early and late recurrences. Early recurrence is mainly driven by aggressive characteristics of the primary (resected) tumor such as tumor size, tumor multiplicity, vascular invasion and higher serum AFP level $[17,19]$. In contrast, late relapse is thought to be primarily due to disease etiology and cirrhotic background (an oncological field change in the liver due to underlying liver disease), both of which are well described risk factors for hepatocarcinogenesis $[10,20]$. Time to recurrence after resection is an important prognostic factor, therefore early HCC recurrence appears to be associated with worse survival rates, compared with those with later recurrence [21,22]. Furthermore, survival rates for patients with early disease recurrence are dependent on the number of risk factors present at the time of surgery [21]. 
As a result of high rates of recurrence following curative resection or ablation, effective and well tolerated adjuvant treatments are urgently needed to prevent or reduce recurrence in order to improve the prognosis of patients with HCC $[1,10]$. Patients at high risk of early HCC recurrence are potential candidates for adjuvant therapy [19]. Many systemic and trans-arterial post operative interventions to reduce recurrence have been studied [23,24], including sorafenib [25] and immunotherapies. However, no intervention has been proven in randomized trials and as a result current mainstream treatment guidelines do not recommend, the use of any adjuvant therapy outside or clinical trials and the standard of care after surgical resection remains close surveillance. Immunotherapeutic approaches have shown some promise in the adjuvant setting, which is of interest when considering studies of other cancer immunotherapy (CIT)-based treatments in the adjuvant setting.

\section{Cancer immunotherapy for HCC}

The advent of CIT, in particular immune checkpoint inhibitors, has transformed cancer treatment across multiple tumor types. Expression of the immune checkpoint molecule PD-L1 on tumor cells and/or tumor-infiltrating immune cells (IC) is known to suppress antitumor immunity. Binding of PD-L1 to its receptor, PD-1, on T cells results in inhibition of proliferation and effector function of $\mathrm{T}$ cells. Atezolizumab is a humanized engineered immunoglobulin G1 monoclonal antibody that selectively targets PD-L1 to block its interaction with PD-1 and the costimulatory molecule B7.1 to reinvigorate tumor-specific T-cell immunity. Phase Ib studies of single-agent PD-L1/PD-1 immune checkpoint inhibitors, including atezolizumab, demonstrated clinical activity in HCC [2628]. However, confirmatory Phase III studies of such single-agent therapies have failed to demonstrate superiority in terms of survival over standard of care treatments [29,30], suggesting that PD-L1/PD-1 axis blockade alone may not be sufficient to initiate adequate levels of anticancer immunity in HCC. These data suggest that combination approaches to target additional immune-suppressive mechanisms may be needed in HCC. The primary goal of combined CIT treatment is to engineer a more favorable environment to maximize the immune system's ability to eliminate cancer. One such approach is to simultaneously inhibit anti-PD-L1 and anti-VEGF pathways. Phase III studies have validated the clinical efficacy of combined PD-1/PD-L1 and VEGF inhibition in advanced RCC and NSCLC [31-33].

\section{Rationale for dual PD-L1 \& VEGF blockade in HCC}

HCC is a hypervascular tumor in which aberrant angiogenesis, driven by growth factors such as VEGF, contributes to tumor growth and metastasis. Over the last decade, treatment for advanced HCC has focused on targeting the VEGF signaling pathway where receptor tyrosine kinase inhibitors, such as sorafenib and lenvatinib, or monoclonal antibodies that block VEGF are standard treatments in the advanced disease setting [34]. In addition to its wellcharacterized role in angiogenesis, VEGF is also believed to play a role in cancer immune evasion (Figure 1). Data from preclinical studies and clinical trials suggest that VEGF can exert immune-suppressive effects via three key mechanisms: downregulating T-cell activation via inhibition of DC maturation, reducing T-cell tumor infiltration and increasing inhibitory cells such as myeloid derived suppressor cells (MDSCs) and regulatory T cells (Tregs) in the tumor microenvironment (TME; Figure 1) [35-37].

Based on the rationale above, the efficacy and tolerability of combined PD-L1 (atezolizumab) and VEGF (bevacizumab) pathway inhibition was evaluated in unresectable HCC in two randomized studies, the results of which have been recently reported (Table 1). These studies sought to answer two primary questions: does VEGF inhibition enhance the efficacy of anti-PD-L1 treatment; and is atezolizumab combined with bevacizumab more effective than standard treatment for unresectable HCC? The first question was addressed by conducting a Phase Ib study (GO30140) that included a randomized arm (Arm F) comparing atezolizumab with atezolizumab plus bevacizumab [27]. The second question was addressed by a Phase III trial (IMbrave 150) comparing atezolizumab plus bevacizumab with sorafenib.

In Arm F of study GO30140, patients with unresectable HCC were randomly assigned 1:1 to receive either atezolizumab alone or atezolizumab in combination with bevacizumab. The primary end point was progression-free survival (PFS) as per RECIST 1.1 assessed by blinded independent review. The results demonstrated that, compared with atezolizumab alone, combination treatment with atezolizumab plus bevacizumab significantly increased PFS (Table 1) [27]. The PFS benefit was consistent across almost all subgroups and methods of tumor assessment (Table 1). These data indicate that anti-VEGF treatment significantly enhances the efficacy of PD-L1 inhibition and combination PD-L1/VEGF blockade is required to augment anticancer immunity in patients with unresectable HCC. 


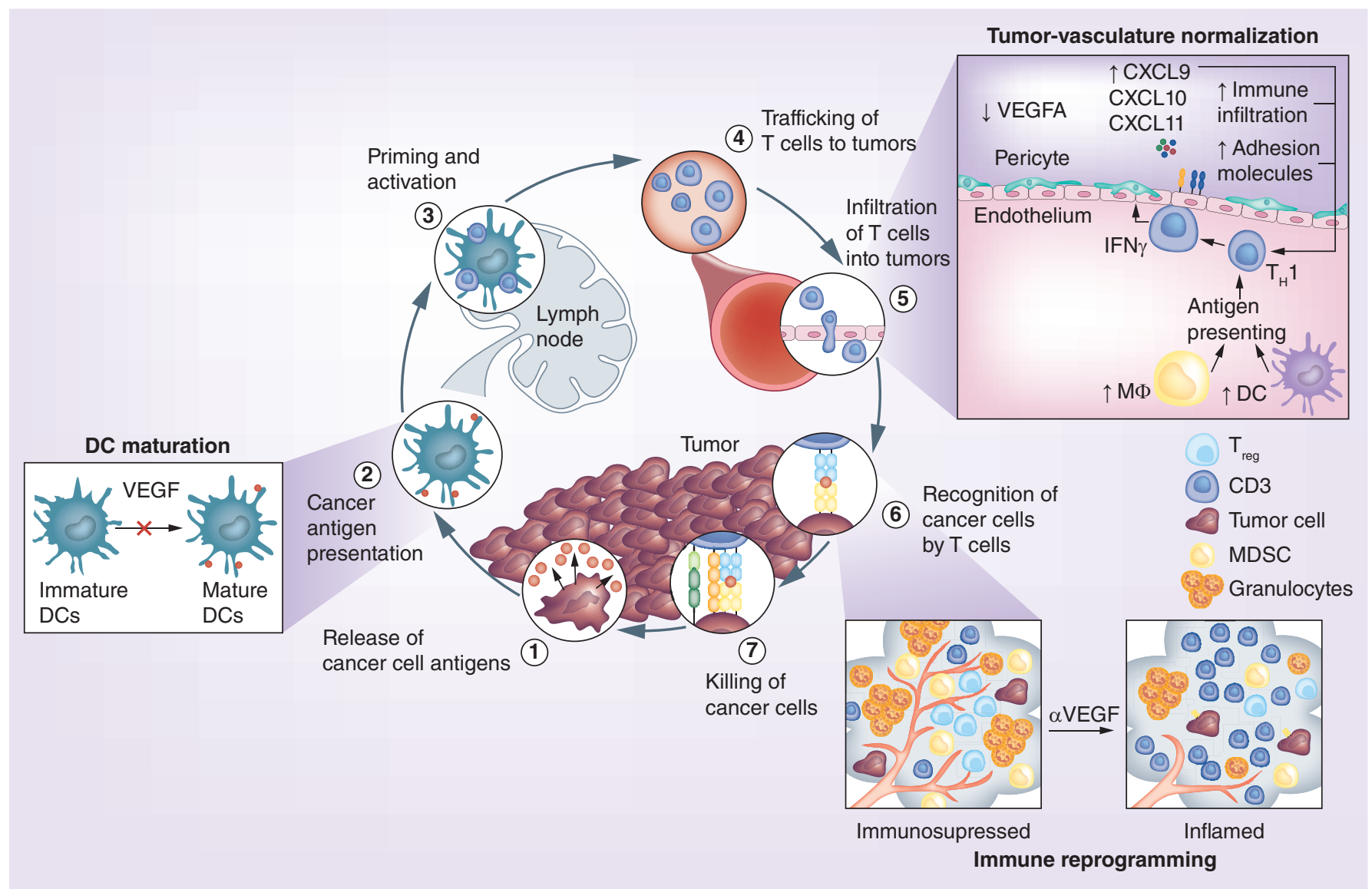

Figure 1. Interaction between VEGF and the cancer immunity cycle.

Reproduced with permission from [36] (c) Elsevier (2018).

\begin{tabular}{|c|c|c|c|c|}
\hline \multirow[t]{2}{*}{ End point } & \multicolumn{2}{|c|}{ G030140: Arm F } & \multicolumn{2}{|c|}{ IMbrave 150} \\
\hline & Atezolizumab $(n=59)$ & $\begin{array}{l}\text { Atezolizumab }+ \\
\text { bevacizumab }(n=60)\end{array}$ & Sorafenib $(n=165)$ & $\begin{array}{l}\text { Atezolizumab }+ \\
\text { bevacizumab }(n=336)\end{array}$ \\
\hline Median duration of follow-up & 6.6 months & 6.7 months & 8.6 months & \\
\hline PFS events, n (\%) & $35(58)$ & $39(66)$ & & \\
\hline Median PFS, months (95\% CI) & $3.4(1.9-5.2)$ & $5.6(3.6-7.4)$ & $4.3(4.0-5.6)$ & $6.8(5.7-8.3)$ \\
\hline Stratified PFS HR (Cl) & $\begin{array}{l}0.55(80 \% \mathrm{Cl}: 0.40-0.74) \\
\mathrm{p}=0.0108\end{array}$ & & $\begin{array}{l}0.59(95 \% \mathrm{Cl}: 0.47-0.76) \\
\mathrm{p} \leq 0.0001\end{array}$ & \\
\hline Median OS, months $(95 \% \mathrm{Cl})$ & $\mathrm{NE}(8.3, \mathrm{NE})$ & $\mathrm{NE}(8.2, \mathrm{NE})$ & $13.2(10.4, \mathrm{NE})$ & NE \\
\hline Stratified OS HR (CI) & 0.78 (80\% Cl: $0.5-1.2)$ & & $\begin{array}{l}0.58(0.42-0.79) \\
p=0.0006\end{array}$ & \\
\hline ORR, \% (95\% Cl) & $17(8-29)$ & $20(11-32)$ & $12(7-18.0)$ & $27(23-33)$ \\
\hline $\begin{array}{l}\text { Difference in ORR } \\
\text { (p-value) }\end{array}$ & $3 \%$ (p-value NR) & & $15 \%(p<0.0001)$ & \\
\hline Ongoing response, $n(\%)$ & $8(80)$ & $12(100)$ & $13(68)$ & $77(87)$ \\
\hline
\end{tabular}

HR: Hazard ratio; ITT: Intent-to-treat; NE: Not estimable; NE: Not Evaluable; NR: Not Reported; NS: Not significant; ORR: Overall response rate; OS: Overall survival; PFS: Progressionfree survival. 


\begin{tabular}{|c|c|c|}
\hline Immune cells/checkpoints & Findings in $\mathrm{HCC}$ & Ref. \\
\hline \multicolumn{3}{|c|}{ Infiltration of immune effectors } \\
\hline Immunoscore & $\uparrow \mathrm{CD}^{+} / \mathrm{CD}^{+}$infiltration associated with $\downarrow$ recurrence & [44] \\
\hline NKT cells \& IFN- $\gamma$ & $\downarrow N K T$ cells and IFN- $\gamma$ independently predict recurrence and survival & [46] \\
\hline Immune gene signatures & Multiple immune gene signatures associated with the prognosis of resected HCC & [47] \\
\hline \multicolumn{3}{|c|}{ Accumulation of immunosuppressive cells } \\
\hline Tregs & $\uparrow$ Tregs associated with $\downarrow$ OS and DFS & $\begin{array}{l}{[48]} \\
{[49]}\end{array}$ \\
\hline MDSCs & $\begin{array}{l}\uparrow \mathrm{MDSCs} \text { correlated with early recurrence after resection } \\
\uparrow \mathrm{PD}-\mathrm{L} 1^{+} \mathrm{MDSCS} \text { in HCC }\end{array}$ & $\begin{array}{l}{[50]} \\
{[51]} \\
{[52]}\end{array}$ \\
\hline Macrophages & $\uparrow$ peritumoral $\mathrm{M} \Phi$ associated with recurrence and poor survival after hepatectomy & $\begin{array}{l}{[53]} \\
{[54]}\end{array}$ \\
\hline \multicolumn{3}{|c|}{ Upregulation of negative immune checkpoints } \\
\hline PD-L1 & $\begin{array}{l}\uparrow \text { PD-L1 associated with high-risk factors for recurrence } \\
\text { Unclear impact on recurrence/survival }\end{array}$ & $\begin{array}{l}{[55]} \\
{[56]} \\
{[57]}\end{array}$ \\
\hline
\end{tabular}

treatment with atezolizumab plus bevacizumab resulted in a significant improvement in both PFS and OS compared with sorafenib (Table 1) [38]. Furthermore, objective response rate assessed either by RECIST 1.1. or mRECIST criteria was significantly higher in patients treated with atezolizumab plus bevacizumab compared with sorafenib (Table 1). The safety profile of atezolizumab plus bevacizumab was consistent with the known safety profile of each individual drug with no new safety signals being identified with the combination.

\section{Why study dual PD-L1/VEGF inhibition in adjuvant HCC?}

Multiple lines of evidence suggest that CIT-based treatments may be promising in the adjuvant HCC setting following curative resection or ablation. In recent years, several CIT modalities have been studied in the adjuvant HCC setting [39]. For over a decade, adoptive immunotherapy - involving the infusion of immune effector cells created by ex vivo expansion of patient's peripheral blood mononuclear cells - have been studied as an adjuvant therapy for HCC. Several randomized studies conducted in Asia have shown that adoptive immunotherapy reduced HCC recurrence and, in some cases, increased OS following curative resection or ablation [40,41]. Small randomized studies have also reported that tumor-directed vaccines can also reduce recurrence rates [42,43]. Together, these studies provide a clinical proof-of-concept for immunotherapy as adjuvant treatment in HCC.

A Phase III trial testing sorafenib (a multikinase inhibitor primarily targeting RAF and VEGF) versus placebo as adjuvant therapy after liver resection or ablation failed to demonstrate any positive effect on either recurrence-free survival (RFS) or OS [25]. This result suggests that targeting angiogenesis alone is insufficient in preventing HCC recurrence and that combinations are likely needed to facilitate effective anticancer immunity.

In addition to clinical factors associated with HCC recurrence such as tumor burden and vascular invasion, immune mechanisms have also been shown to be associated with recurrence (Table 2). The immune contexture of the TME in the liver is thought to play a critical role in determining HCC outcome, including recurrence risk after resection or ablation. For the most part, studies of resected HCC specimens have shown that infiltration by immune effector cells such as T cells, $\mathrm{CD} 8^{+} \mathrm{T}$ cells, $\mathrm{T} \gamma \delta$ cells and NK cells is associated with a lower risk of post operative recurrence compared with cases with lower levels of IC infiltration. Using the Immunoscore - a composite assay incorporating density of $\mathrm{CD}^{+}$and $\mathrm{CD}^{+} \mathrm{T}$ cells in the interior and margin of the tumor - Gabrielson et al. demonstrated that a high immunoscore was associated with a markedly reduced rate of HCC recurrence and prolonged RFS [44]. This suggests that $\mathrm{CD}^{+}$and $\mathrm{CD}^{+}$positive T-cell populations infiltrating the tumor play an important role in generating the anticancer immune response mediating the prevention of HCC recurrence. Infiltration of other cytotoxic T cells, such as $\mathrm{CD}^{+}$, is also linked with lower recurrence risk [45]. Trafficking of primed and activated $\mathrm{T}$ cells from the lymph node into circulation and then to the tumor requires a series of steps, many of which are regulated by VEGF. Preclinical and clinical data have shown that immunosuppressive effects of 
VEGF are, to a significant extent, mediated by abnormal tumor vasculature that is induced by VEGF, which in turn can prevent tumoral T-cell infiltration and promote tumor immune escape [35].

The accumulation of immune-suppressive cell types such as MDSCs and Tregs has also been linked to a high rate of HCC recurrence. MDSCs mediate both angiogenesis and immunosuppression in the TME. The mechanisms by which MDSCs mediate immunosuppression in the TME are thought to include: inducing differentiation and expansion of Tregs; inhibiting the polarization of DCs, NKs and macrophages to the M2 phenotype; depriving $\mathrm{T}$ cells of essential amino acids; inducing oxidative stress; and VEGF overexpression [58]. Multiple studies have shown that MDSCs are increased in the peripheral blood of patients with HCC compared with healthy controls and patients with cirrhosis or hepatitis. Moreover, a high frequency of MDSCs in PBMCs has been associated with aggressive tumor features and poor clinical outcomes after hepatectomy or local ablation [59,60]. PD-L1 ${ }^{+}$MDSCs are increased in both peripheral blood and tumor-infiltrating lymphocytes in patients with HCC which were reduced after curative treatment and demonstrated an inverse association with DFS after resection [52]. Notably, PD-L1 ${ }^{+}$ MDSCs were strongly induced in liver cancer cell lines by VEGF and their proliferation could be potently reduced by a VEGF-neutralizing antibody [52]. Interestingly, a randomized Phase II study in RCC, an immunosuppressed angiogenic cancer, demonstrated that combined PD-L1/VEGF inhibition with atezolizumab and bevacizumab was particularly effective in RCC with a myelosuppressive phenotype [61]. In addition to MDSCs, Tregs are an immunosuppressive cell type that are elevated in HCC compared with healthy liver and are linked with poor DFS [48,62]. Both Tregs, MDSCs and inhibitory checkpoints such as PD-1 have been shown to act in a cooperative fashion to cause immunosuppression in the HCC TME [63]. Supporting the idea that immune networks and the balance of immune effector/immune suppressive cell are critical mediators of recurrence, the ratio of Tregs and cytotoxic $\mathrm{T}$ cells is an independent predictor for recurrence and survival following resection [49]. Along with studies of infiltrating IC types, several immune gene signatures have characterized the TME of resected HCC specimens and have shown that immunogenic signatures are associated with lower rates of recurrence and/or longer survival following resection $[47,64,65]$. Multiple studies involving an immune signature have reported that while around $25 \%$ of early-stage HCC have a phenotype that would likely derive benefit from a PD-1/PD-L1 inhibitor, most harbor immunosuppressive features that would require combination CIT approaches to facilitate effective anticancer immunity.

Ablation, in particular RFA, can induce a variety of immunologic effects. These include release of tumor antigens as a result of thermally induced necrosis, triggering of inflammatory cytokines at the sub-lethal zone surrounding the necrotic zone of the ablated area and upregulation of cytotoxic T-cell subsets [66]. RFA has been shown to enhance various tumor-associated antigen-specific T-cell responses for several weeks following ablation, with the number of $\mathrm{T}$ cells induced being associated RFS [67]. Interestingly, the number of tumor-associated antigens was inversely correlated with the frequency of MDSCs, suggesting myeloid-mediated mechanisms may be operating following ablation [67]. While ablation can induce favorable immunogenic effects, it appears that these changes are insufficient to ultimately prevent recurrence. This suggests that CIT regimens may help to amplify favorable immune mechanisms within the TME to prolong or prevent recurrence following ablation.

Immunosuppressive mechanisms within the TME such as reduced T-cell infiltration, upregulation of immune checkpoints and expansion of immune suppressive cell types such as MDSCs and Tregs have been shown to contribute to HCC recurrence following either resection or ablation. All of these mechanisms are thought to be modulated by VEGF $[35,65]$ and it is reasonable to postulate that the VEGF-induced immunosuppression plays a critical role in the TME of HCC and is a driver of HCC recurrence after curative treatment.

Taken together, the following rationale can be proposed to study combined PD-L1/VEGF inhibition in the adjuvant HCC setting:

- Immunotherapeutic approaches have shown promise in reducing recurrence rates after resection or ablation;

- The immune contexture of the TME in the liver plays a critical role in determining recurrence risk after resection or ablation. Only approximately $25 \%$ of early-stage HCCs have genomic evidence of immune activation;

- Randomized studies in unresectable/advanced HCC have shown that: combined anti-PD-L1/VEGF with atezolizumab and bevacizumab is superior to atezolizumab monotherapy; atezolizumab plus bevacizumab significantly increases OS, PFS and objective response rate compared with sorafenib (a VEGF TKI); PD-1 inhibition alone is not sufficient to improve survival; 


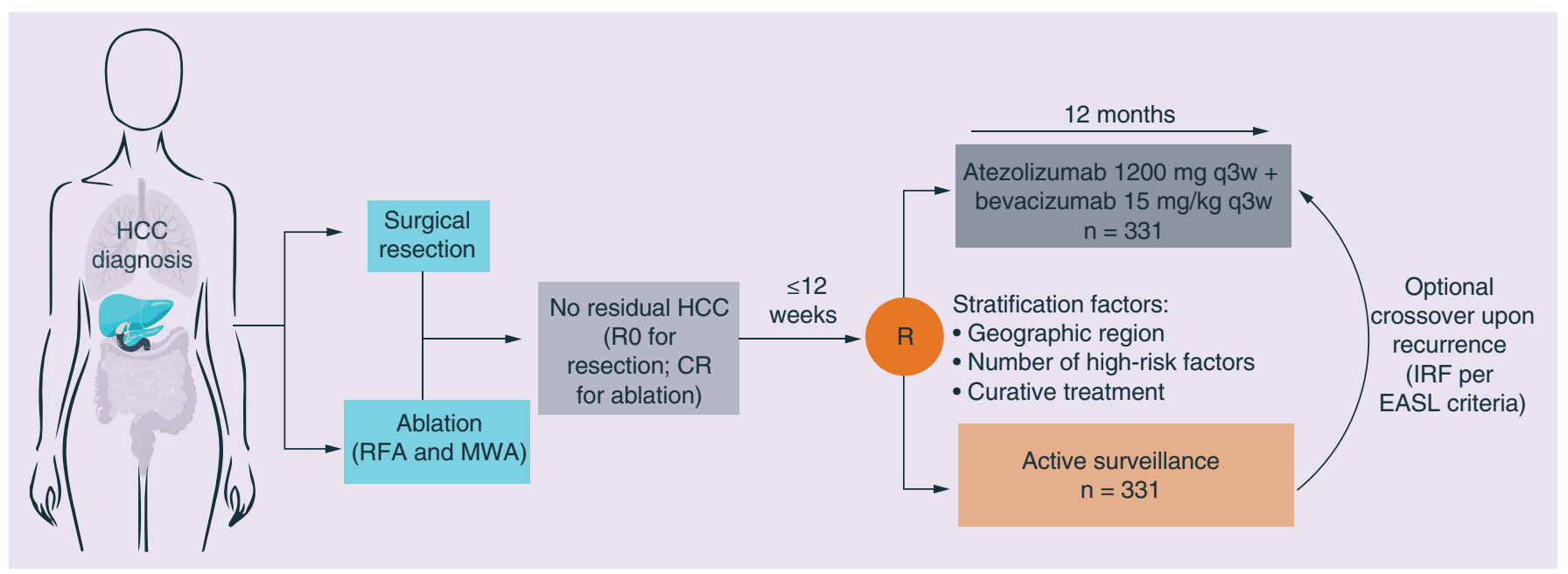

Figure 2. IMbrave 050 study design. Diagnosis of HCC by radiological criteria and/or pathological confirmation. CR: Complete response by imaging; EASL: European Association for the Study of the Liver; HCC: Hepatocellular carcinoma; IRF: Independent review facility; MWA: Microwave ablation; q3w: Every 3 weeks; R0: Absence of microscopic tumor invasion of the resection margin; RFA: Radiofrequency ablation; R: Randomization.

- Many of the immune mechanisms underlying immunosuppression in the liver TME that are associated with HCC recurrence for example reduced infiltration of cytotoxic T cells, upregulation of immune checkpoints and accumulation of immune-suppressive cell types such as MDSCs and Tregs are regulated by VEGF.

\section{The IMbrave 050 study}

Here, we describe the design of IMbrave 050, a Phase III, randomized, multicenter, open-label study of atezolizumab plus bevacizumab versus active surveillance in patients at high risk of disease recurrence following curative resection or ablation. The study is funded by F. Hoffmann-La Roche Ltd.

\section{Study design}

Approximately 662 patients who have undergone either surgical resection or ablation and who are at high-risk of HCC recurrence will be randomized in a 1:1 ratio to either atezolizumab plus bevacizumab or active surveillance (Figure 2). Randomization will be stratified according to geographic region (Asia Pacific region or Rest of World), number of high-risk features (single or multiple), curative procedure and use of adjuvant TACE. High-risk features for resected patients include tumor size $>5 \mathrm{~cm}$, tumor number $>3$, vascular invasion (microvascular invasion or macrovascular invasion - Vp1/Vp2 - of the portal vein) and poor tumor differentiation (defined as Grade 3 or 4). Resected patients will therefore have between 1 and 4 high-risk factors. Randomization must occur within 12 weeks following resection or ablation. IMbrave 050 is being conducted at approximately 170 sites in 25 countries with the goal of recruiting 662 patients. Recruitment will be competitive.

\section{Patient population}

Key eligibility criteria are described in Box 1 . All patients (men or women) are 18 years or older with a first diagnosis of HCC who have undergone either definitive resection or ablation (by either RFA or microwave ablation). Resected patients are required to have histological confirmation of negative surgical margins ( $\mathrm{R} 0$ or equivalent), while ablated patients require documentation of a complete radiological response. Only patients at high-risk of HCC recurrence based on tumor characteristics derived from pre procedure imaging and post operative histopathology (resection only) will be eligible. The definition of high recurrence risk is based on composite criteria that includes tumor size, tumor number, presence of microvascular invasion or minor macrovascular invasion of the portal vein $(\mathrm{Vp} 1$ or $\mathrm{Vp} 2$ ) and/or poorly differentiated microscopic appearance (histologic Grade 3 or 4). The criteria for high risk of HCC recurrence used in this study is presented by type of curative treatment in Table 3. For patients who have undergone ablation, recurrence risk is defined by tumor burden (size and number) only (Table 3). Definitions of microvascular and macrovascular invasion as they relate to eligibility for IMbrave 050 are shown in Table 4 . The protocol does not stipulate the criteria to be used to assess histologic grading because of differences in institutional 
Box 1. IMbrave 050 eligibility criteria.

Inclusion criteria

- Age $\geq 18$ years

- Participants with a first diagnosis of HCC by radiological criteria and/or pathological confirmation, who have undergone a curative resection or ablation (RFA or MWA only)

- Documented diagnosis of HCC that has been completely resected or ablated (RFA or MWA only) as described below:

- Patients with resected HCC must have documented histological confirmation of negative surgical margins (R0);

- Patients with ablated HCC must have documented evidence of complete radiological response, including disappearance of any intratumoral arterial enhancement in all ablated lesions;

- All patients must have disease-free status documented within 4 weeks prior to randomization.

- Absence of major macrovascular (gross vascular) invasion of the portal vein ( $\mathrm{Vp} 3$ or $\mathrm{Vp} 4$ ) or any grade of macrovascular invasion in the hepatic vein or inferior vena cava.

- Note: patients with minor vascular invasion of the portal vein ( $\mathrm{Vp} 1$ or $\mathrm{Vp} 2)$ as detected by either imaging or pathological examination are allowed.

- No extrahepatic HCC

- Full recovery from surgical resection or ablation within 4 weeks prior to randomization

- High risk for HCC recurrence after resection or ablation

- Full recovery from adjuvant TACE procedure within 4 weeks prior to randomization

- Willingness to provide a baseline tumor tissue sample (resected patients only)

- Documented virology status of hepatitis, as confirmed by screening HBV and HCV tests

- For patients with active HBV:

- HBV DNA <500 IU/ml during screening;

- initiation of anti-HBV treatment at least 14 days prior to randomization and willingness to continue anti-HBV treatment during the study.

- Patients with HCV, either with resolved infection (as evidenced by detectable antibody) or chronic infection (as evidenced by detectable HCV RNA), are eligible

- Performance of an esophagogastroduodenoscopy either before resection or ablation or during screening and assessment and treatment of varices of all sizes per local standard of care prior to randomization

- ECOG Performance Status of 0 or 1

- Child-Pugh Class A status

- Adequate hematologic and end-organ function defined by laboratory test results

Exclusion criteria

- Recurrent HCC prior to randomization

- Clinically significant ascites

- History of hepatic encephalopathy

- Prior bleeding event due to untreated or incompletely treated esophageal and/or gastric varices within 6 months prior to randomization

- Active or history of autoimmune disease or immune deficiency

- Significant cardiovascular disease (such as New York Heart Association Class II or greater cardiac disease, myocardial infarction or cerebrovascular accident) within 3 months prior to Day 1 of Cycle 1, unstable arrhythmia or unstable angina

- On the waiting list for liver transplantation

- Coinfection with HBV and HCV

- Patients with a history of HCV infection but who are negative for HCV RNA by PCR will be considered to be negative for HCV infection

- Any treatment for HCC prior to resection or ablation, including systemic therapy (including investigational agents) and locoregional therapy such as TACE

- Prior use of herbal therapies or traditional Chinese medicines with anticancer activity included in the label is allowed, but such therapies must be discontinued at least 7 days prior to randomization and are prohibited during the study

- Prior treatment with CD137 agonists or immune checkpoint blockade therapies, including anti-CTLA-4, anti-PD-1 and anti-PD-L1 therapeutic antibodies

- Inadequately controlled arterial hypertension (defined as systolic blood pressure [BP] $>150 \mathrm{mmHg}$ and/or diastolic BP $>100 \mathrm{mmHg}$ ), based on an average of at least three BP readings at two or more sessions

- Antihypertensive therapy to achieve these parameters is allowed

- History of hypertensive crisis or hypertensive encephalopathy

- Significant vascular disease (e.g., aortic aneurysm requiring surgical repair or recent peripheral arterial thrombosis) within 6 months prior to Day 1 of Cycle 1

HCC: Hepatocellular carcinoma; MWA: Microwave ablation; RFA: Radiofrequency ablation. 
Table 3. Criteria for high risk of hepatocellular carcinoma recurrence.

\begin{tabular}{|c|c|}
\hline Curative treatment & Criteria \\
\hline Resection & $\begin{array}{l}\text { Up to three tumors, with largest tumor }>5 \mathrm{~cm} \text { regardless of vascular invasion (microvascular invasion or macrovascular } \\
\text { invasion of } \mathrm{Vp} 1 / \mathrm{Vp} 2 \text { ) or poor tumor differentiation (Grade } 3 \text { or } 4 \text { ) } \\
\text { Four or more tumors, with largest tumor } \leq 5 \mathrm{~cm} \text { regardless of vascular invasion (microvascular invasion or macrovascular } \\
\text { invasion of } \mathrm{Vp} 1 / \mathrm{Vp} 2 \text { ) or poor tumor differentiation (Grade } 3 \text { or } 4 \text { ) } \\
\text { Up to three tumors, with largest tumor } \leq 5 \mathrm{~cm} \text { with vascular invasion (microvascular invasion or macrovascular invasion of } \\
\mathrm{Vp} 1 / \mathrm{Vp} 2 \text { ) and/or poor tumor differentiation (Grade } 3 \text { or } 4 \text { ) }\end{array}$ \\
\hline Ablation & $\begin{array}{l}\text { Single tumor }>2 \mathrm{~cm} \text { but } \leq 5 \mathrm{~cm} \\
\text { Multiple tumors (up to four tumors), all } \leq 5 \mathrm{~cm}\end{array}$ \\
\hline
\end{tabular}

\section{Table 4. Definition of types of vascular invasion in hepatocellular carcinoma.}

\begin{tabular}{|c|c|c|}
\hline Type of vascular invasion & Definition & Eligible for IMbrave 050 \\
\hline \multicolumn{3}{|l|}{ Portal vein } \\
\hline Vpo & No evidence of tumor thrombus invasion & Yes \\
\hline Vp1 & Tumor thrombus distal to but not in the second-order branches & Yes \\
\hline Vp2 & Tumor thrombus in the second-order branches & Yes \\
\hline Vp3 & Tumor thrombus in the first-order branches & No \\
\hline Vp4 & Tumor thrombus in the main trunk or contralateral or both & No \\
\hline \multicolumn{3}{|l|}{ Hepatic vein } \\
\hline Vv0 & Absence of tumor thrombus invasion of the hepatic vein & Yes \\
\hline Vv1 & Tumor thrombus invasion of peripheral branches of the hepatic vein & No \\
\hline Vv2 & $\begin{array}{l}\text { Tumor thrombus invasion of the right, middle or left hepatic vein, the inferior right } \\
\text { hepatic vein, or the short hepatic vein }\end{array}$ & No \\
\hline Vv3 & Tumor thrombus invasion of the inferior vena cava & No \\
\hline \multicolumn{3}{|l|}{ Microvascular invasion } \\
\hline $\mathrm{N} / \mathrm{A}$ & $\begin{array}{l}\text { Presence of microscopic thrombi within the central hepatic vein, the portal vein or large } \\
\text { capsular vessels }\end{array}$ & Yes \\
\hline
\end{tabular}

practice and the broad similarity of available criteria [68]. Up to one cycle of TACE may be administered following resection at the discretion of the investigator. Use of adjuvant TACE is commonly used in China for patients at high risk of recurrence and is included in Chinese treatment guidelines [69]. Patients are required to have fully recovered from the curative procedure as well as any adjuvant TACE at least 4 weeks prior to randomization. Key exclusion criteria are listed in Box 1.

\section{Study procedures \& treatment}

Patients randomized to the treatment arm will be given atezolizumab (1200 mg every 3 weeks iv.) and bevacizumab (15 mg/kg every 3 weeks iv.). This regimen will be given for a period of 1 year or approximately 17 cycles unless disease recurrence or unacceptable toxicity occurs. Patients randomized to the control arm will undergo active surveillance which represents the standard of care following complete surgical resection or ablation [10]. Imaging will be performed every 12 weeks following randomization for the first 3 years and then every 24 weeks thereafter until disease recurrence or until the end of Year 7. Intrahepatic recurrence of HCC will be assessed according to EASL criteria based on imaging and/or histopathologic confirmation [10]. Extrahepatic recurrence will be defined as per RECIST 1.1 criteria [70].

Patients randomized to the control arm will be allowed to crossover to combination treatment with atezolizumab and bevacizumab following confirmation of recurrence by the independent review facility at the discretion of the investigator. The option to crossover to atezolizumab plus bevacizumab will be offered to patients randomized to the control arm as either adjuvant treatment for patients who undergo a second resection or as systemic treatment for patients indicated for systemic treatment following recurrence.

Patient Reported Outcomes assessments will be performed every six weeks after randomization for patients in both arms. An independent Data Monitoring Committee will review safety at regular intervals and efficacy for the purposes of the first interim analysis. 
Box 2. IMbrave 050 study end points.

Primary end point

- RFS by IRF

Key secondary end points

- OS

- TTR

- RFS as determined by the investigator

- IRF-assessed RFS and investigator-assessed RFS rate at 24 and 36 months after randomization

- OS rate at 24 and 36 months

- Time to EHS or MVI

- RFS for patients in the PD-L1-high subgroup

- Safety

Key exploratory end points

- RFS or PFS for crossover patients (by investigator assessment)

- ORR for crossover patients (RECIST v1.1)

- Relationship between blood- or tissue-based biomarkers and efficacy

EASL: European Association for the Study of the Liver; EHS: Extrahepatic spread; IRF: Independent Review Facility; MVI: Macrovascular invasion; ORR: Objective response rate; OS: Overall survival; PFS: Progression-free survival; TTR: Time to recurrence; RFS: Recurrence-free survival.

Study end points

The primary end point of IMbrave 050 is RFS assessed by a blinded independent review facility. RFS is defined as the time from randomization to the first documented occurrence of local, regional or metastatic HCC or death from any cause (whichever occurs first). Other key secondary and exploratory end points are listed in Box 2. RFS analysis will be performed based on the ITT population using the Kaplan-Meier method to estimate median RFS for each arm. A stratified Cox proportional-hazards model will be used to estimate the HR and its 95\% CI. It is acknowledged that the option for control arm patients to crossover to atezolizumab plus bevacizumab may compromise the ability to demonstrate an OS benefit.

\section{Conclusion}

An immunosuppressive TME is a critical determinant of HCC recurrence following curative intervention. Many of the mechanisms contributing to hepatic immune suppression are regulated by VEGF as well as inhibitory immune checkpoints such as PD-1/PD-L1. IMbrave 150, a Phase III study comparing atezolizumab (a PD-L1 inhibitor) and bevacizumab (a VEGF inhibitor) to sorafenib in unresectable HCC, demonstrated that combination PD-L1/VEGF blockade significantly increased OS, PFS and response rate. It is hypothesized that concurrent PD-L1 and VEGF inhibition may be effective in reducing HCC recurrence by creating a more immune-favorable microenvironment, thereby enhancing anticancer immunity. In order to test this hypothesis, we designed the IMbrave 050 study described herein to evaluate atezolizumab (a PD-L1 inhibitor) combined with bevacizumab (a VEGF inhibitor) for HCC at high risk of recurrence following curative resection or ablation. Enrollment began in December 2019 and the estimated study completion date is March 2023. 
- Liver resection and ablation are important curative-intent treatment options for patients with hepatocellular carcinoma (HCC).

- Despite definitive resection or ablation, patients experience high rates of disease recurrence $(70-80 \%$ recurrence at 5 years).

- No adjuvant treatments are currently recommended in treatment guidelines and effective treatments that decrease the incidence of HCC recurrence are urgently needed.

Rationale for cancer immunotherapy as adjuvant therapy

- The contexture of the immune microenvironment in the liver is an important determinant of HCC recurrence.

- Many immunosuppressive mechanisms present in the tumor microenvironment that are implicated in HCC recurrence are modulated by VEGF and/or PD-L1.

- IMbrave 150, a randomized Phase III trial, has shown that atezolizumab (a PD-L1 inhibitor) plus bevacizumab (a VEGF inhibitor) significantly improves overall survival, progression-free survival and overall response rate in patients with unresectable HCC.

- Dual PD-L1/VEGF blockade may be effective in reducing HCC recurrence by creating a more immune-permissive microenvironment, thereby enhancing anticancer immunity.

IMbrave 050

- This multinational, randomized (1:1), two-arm, open-label trial is evaluating atezolizumab combined with bevacizumab versus active surveillance for $\mathrm{HCC}$ at high risk of recurrence after curative resection or ablation.

- The primary end point is recurrence-free survival.

\section{Supplementary data}

An infographic accompanies this paper at the end of the references section. To download the infographic that accompanies this paper, please visit the journal website at: www.futuremedicine.com/doi/10.2217/fon-2020-0162

\section{Acknowledgments}

The authors thank the patients participating in the trial and their families, the investigators and staff at participating centers, the independent Data Monitoring Committee and the study team at F Hoffmann-La Roche Ltd. The authors also wish to thank M Kowgier for his statistical expertise in designing IMbrave 050.

Financial \& competing interests disclosure

SP Hack is a full-time employee of Roche/Genentech and holds Roche stock. J Spahn is a full-time employee of Roche/Genentech and holds Roche stock. A-L Cheng has received honoraria from AstraZeneca, Bayer Yakuhin, Eisai, Genentech/Roche and Lilly and consulting/advisory fees from AstraZeneca, Bayer Schering Pharma, BeiGene, Bristol-Myers Squibb, CSR Pharma Group, Eisai, Genentech/Roche, MSD, Novartis and Ono Pharmaceutical. M Kudo is a consultant of Bayer, Eisai, Ono, MSD, BMS, Eli Lilly and received research grant from Ono, BMS, MSD, Eisai, and received honoraria from Bayer, Eisai and BMS. HC Lee has received grants from Bayer, Bristol-Myers Squibb, Roche, MSD, AstraZeneca and Sillajen. P Chow has received honoraria and has participated in advisory boards for Sirtex Medical, Ipsen, BMS, Oncosil, Bayer, Roche, New B Innovation, MSD and BTG PLC. P Chow has received research funding support from Sirtex Medical, Ipsen, IQVIA, New B Innovation, MSD, Perspectum Diagnostic and Genentech. The authors have no other relevant affiliations or financial involvement with any organization or entity with a financial interest in or financial conflict with the subject matter or materials discussed in the manuscript apart from those disclosed.

No writing assistance was utilized in the production of this manuscript.

\section{Open access}

This work is licensed under the Attribution-NonCommercial-NoDerivatives 4.0 Unported License. To view a copy of this license, visit http://creativecommons.org/licenses/by-nc-nd/4.0/

\section{References}

Papers of special note have been highlighted as: $\bullet$ of interest; $\bullet \bullet$ of considerable interest

1. Villanueva A. Hepatocellular Carcinoma. N. Engl. J. Med. 380(15), 1450-1462 (2019).

2. Forner A, Reig M, Bruix J. Hepatocellular carcinoma. Lancet 391(10127), 1301-1314 (2018).

3. Rich NE, Yopp AC, Singal AG. Medical management of hepatocellular carcinoma. J. Oncol. Pract. 13(6), 356-364 (2017). 
4. Kinoshita A, Onoda H, Fushiya N, Koike K, Nishino H, Tajiri H. Staging systems for hepatocellular carcinoma: current status and future perspectives. World J. Hepatol. 7(3), 406-424 (2015).

5. Yau T, Tang VY, Yao TJ, Fan ST, Lo CM, Poon RT. Development of Hong Kong liver cancer staging system with treatment stratification for patients with hepatocellular carcinoma. Gastroenterology 146(7), 1691-1700 (2014).

6. Forner A, Llovet JM, Bruix J. Hepatocellular carcinoma. Lancet 379(9822), 1245-1255 (2012).

7. Omata M, Cheng AL, Kokudo $\mathrm{N}$ et al. Asia-Pacific clinical practice guidelines on the management of hepatocellular carcinoma: a 2017 update. Hepatol. Int. 11(4), 317-370 (2017).

8. Torzilli G, Belghiti J, Kokudo $\mathrm{N}$ et al. A snapshot of the effective indications and results of surgery for hepatocellular carcinoma in tertiary referral centers: is it adherent to the EASL/AASLD recommendations?: an observational study of the HCC East-West study group. Ann. Surg. 257(5), 929-937 (2013).

9. Li ZL, Yu JJ, Guo JW et al. Liver resection is justified for multinodular hepatocellular carcinoma in selected patients with cirrhosis: a multicenter analysis of 1,066 patients. Eur. J. Surg. Oncol. 45(5), 800-807 (2019).

10. European Association for the Study of the Liver. EASL Clinical Practice Guidelines: management of hepatocellular carcinoma. J. Hepatol. 69(1), 182-236 (2018).

11. Heimbach JK, Kulik LM, Finn RS et al. AASLD guidelines for the treatment of hepatocellular carcinoma. Hepatology 67(1), 358-380 (2018).

12. Liang $L$, Xing $H$, Zhang $H$ et al. Surgical resection versus transarterial chemoembolization for BCLC intermediate stage hepatocellular carcinoma: a systematic review and meta-analysis. HPB 20(2), 110-119 (2018).

13. Farinati F, Vanin V, Giacomin A et al. BCLC stage B hepatocellular carcinoma and transcatheter arterial chemoembolization: a 20-year survey by the Italian Liver Cancer group. Liver Int. 35(1), 223-231 (2015).

14. Lim KC, Chow PKH, Allen JC, Siddiqui FJ, Chan ESY, Tan SB. Systematic review of outcomes of liver resection for early hepatocellular carcinoma within the Milan criteria. Br. J. Surg. 99(12), 1622-1629 (2012).

15. Zhong JH, Rodriguez AC, Ke Y, Wang YY, Wang L, Li LQ. Hepatic resection as a safe and effective treatment for hepatocellular carcinoma involving a single large tumor, multiple tumors, or macrovascular invasion. Medicine 94(3), e396 (2015).

16. Li L, Zhang J, Liu X, Li X, Jiao B, Kang T. Clinical outcomes of radiofrequency ablation and surgical resection for small hepatocellular carcinoma: a meta-analysis. J. Gastroenterol. Hepatol. 27(1), 51-58 (2012).

17. Imamura $\mathrm{H}$, Matsuyama $\mathrm{Y}$, Tanaka $\mathrm{E}$ et al. Risk factors contributing to early and late phase intrahepatic recurrence of hepatocellular carcinoma after hepatectomy. J. Hepatol. 38(2), 200-207 (2003).

18. Aufhauser DD Jr, Sadot E, Murken DR et al. Incidence of occult intrahepatic metastasis in hepatocellular carcinoma treated with transplantation corresponds to early recurrence rates after partial hepatectomy. Ann. Surg. 267(5), 922-928 (2018).

19. Chan AWH, Zhong J, Berhane $S$ et al. Development of pre and post-operative models to predict early recurrence of hepatocellular carcinoma after surgical resection. J. Hepatol. 69(6), 1284-1293 (2018).

-. Represents the largest study to date (3903 patients who underwent curative hepatic resection at six medical centers in Asia and the West) that identifies key risk factors for post resection recurrence in patients with hepatocellular carcinoma (HCC).

20. Utsunomiya T, Shimada M, Kudo M et al. A comparison of the surgical outcomes among patients with HBV-positive, HCV-positive, and Non-B Non-C hepatocellular carcinoma: a nationwide study of 11,950 patients. Ann. Surg. 261(3), 513-520 (2015).

21. Jung S-M, Kim JM, Choi G-S et al. Characteristics of early recurrence after curative liver resection for solitary hepatocellular carcinoma. J. Gastrointest. Surg. 23(2), 304-311 (2019).

22. Tabrizian P, Jibara G, Shrager B, Schwartz M, Roayaie S. Recurrence of hepatocellular cancer after resection: patterns, treatments, and prognosis. Ann. Surg. 261(5), 947-955 (2015).

23. Lu LC, Cheng AL, Poon RT. Recent advances in the prevention of hepatocellular carcinoma recurrence. Semin. Liver Dis. 34(4), 427-434 (2014).

24. Zhong JH, Zhong QL, Li LQ, Li H. Adjuvant and chemopreventive therapies for resectable hepatocellular carcinoma: a literature review. Tumour Biol. 35(10), 9459-9468 (2014).

25. Bruix J, Takayama T, Mazzaferro V et al. Adjuvant sorafenib for hepatocellular carcinoma after resection or ablation (STORM): a Phase III, randomised, double-blind, placebo-controlled trial. Lancet Oncol. 16(13), 1344-1354 (2015).

26. El-Khoueiry AB, Sangro B, Yau T et al. Nivolumab in patients with advanced hepatocellular carcinoma (CheckMate 040): an open-label, non-comparative, Phase I/II dose escalation and expansion trial. Lancet 389(10088), 2492-2502 (2017).

27. Lee M, Ryoo BY, Hsu CH et al. LBA39Randomised efficacy and safety results for atezolizumab (Atezo)+bevacizumab (Bev) in patients (pts) with previously untreated, unresectable hepatocellular carcinoma (HCC). Ann. Oncol. 30(Suppl.5), v875 (2019).

-• First randomized study in HCC evaluating the efficacy and safety of combining a VEGF inhibitor (bevacizumab) with an anti-PD-L1 agent (atezolizumab) compared to atezolizumab alone. Demonstrates that combination treatment was superior to atezolizumab alone in terms of progression-free survival (PFS). 
28. Zhu AX, Finn RS, Edeline J et al. Pembrolizumab in patients with advanced hepatocellular carcinoma previously treated with sorafenib (KEYNOTE-224): a non-randomised, open-label Phase II trial. Lancet Oncol. 19(7), 940-952 (2018).

29. Finn RS, Ryoo BY, Merle P et al. Pembrolizumab as second-line therapy in patients with advanced hepatocellular carcinoma in KEYNOTE-240: a randomized, double-blind, Phase III trial. J. Clin. Oncol. 38(3), 192-202 (2019)

- Demonstrates that single-agent pembrolizumab did not significantly improve overall survival (OS) or PFS compared with placebo in patients with advanced HCC who had been previously treated with sorafenib. Overall response rate was significantly higher in the pembrolizumab arm highlighting the antitumor activity of antiprogrammed death-1/PD-L1 agents in HCC.

30. Yau T, Park JW, Finn RS et al. LBA38_PRCheckMate 459: a randomized, multi-center Phase III study of nivolumab (NIVO) vs sorafenib (SOR) as first-line (1L) treatment in patients (pts) with advanced hepatocellular carcinoma (aHCC). Ann. Oncol. 30(Suppl.5), v874 (2019).

-. Shows that single-agent nivolumab did not significantly improve OS compared with sorafenib in patients with advanced HCC.

31. Motzer RJ, Penkov K, Haanen J et al. Avelumab plus axitinib versus sunitinib for advanced renal-cell carcinoma. N. Engl. J. Med. 380(12), 1103-1115 (2019).

32. Socinski MA, Jotte RM, Cappuzzo F et al. Atezolizumab for first-line treatment of metastatic nonsquamous NSCLC. N. Engl. J. Med. 378(24), 2288-2301 (2018).

33. Rini BI, Plimack ER, Stus V et al. Pembrolizumab plus axitinib versus sunitinib for advanced renal-cell carcinoma. N. Engl. J. Med. 380(12), 1116-1127 (2019).

34. Llovet JM, Montal R, Sia D, Finn RS. Molecular therapies and precision medicine for hepatocellular carcinoma. Nat. Rev. Clin. Oncol. 15(10), 599-616 (2018).

35. Chen DS, Hurwitz H. Combinations of bevacizumab with cancer immunotherapy. Cancer J. 24(4), 193-204 (2018).

36. Hegde PS, Wallin JJ, Mancao C. Predictive markers of anti-VEGF and emerging role of angiogenesis inhibitors as immunotherapeutics. Semin. Cancer Biol. 52, 117-124 (2018).

37. Fukumura D, Kloepper J, Amoozgar Z, Duda DG, Jain RK. Enhancing cancer immunotherapy using antiangiogenics: opportunities and challenges. Nat. Rev. Clin. Oncol. 15(5), 325-340 (2018).

38. Cheng A-L, Qin S, Ikeda M et al. LBA3IMbrave150: efficacy and safety results from a ph III study evaluating atezolizumab (atezo) + bevacizumab (bev) vs sorafenib (Sor) as first treatment (tx) for patients (pts) with unresectable hepatocellular carcinoma (HCC). Ann. Oncol. 30(Suppl.9), ix186 (2019).

-• First randomized Phase III study demonstrates superior efficacy (OS, PFS and overall response rate) of cancer immunotherapy (atezolizumab combined with bevacizumab) over sorafenib for advanced HCC. Clinical validation for dual PD-L1/VEGF blockade in HCC

39. Brown ZJ, Greten TF, Heinrich B. Adjuvant treatment of hepatocellular carcinoma: prospect of immunotherapy. Hepatology 70(4), 1437-1442 (2019).

40. Takayama T, Sekine T, Makuuchi M et al. Adoptive immunotherapy to lower postsurgical recurrence rates of hepatocellular carcinoma: a randomised trial. Lancet 356(9232), 802-807 (2000).

41. Lee JH, Lee JH, Lim YS et al. Adjuvant immunotherapy with autologous cytokine-induced killer cells for hepatocellular carcinoma. Gastroenterology 148(7), 1383-1391.e1386 (2015).

42. Peng BG, Liang LJ, He Q et al. Tumor vaccine against recurrence of hepatocellular carcinoma. World J. Gastroenterol. 11(5), 700-704 (2005).

43. Kuang M, Peng BG, Lu MD et al. Phase II randomized trial of autologous formalin-fixed tumor vaccine for postsurgical recurrence of hepatocellular carcinoma. Clin. Cancer Res 10(5), 1574 (2004).

44. Gabrielson A, Wu Y, Wang H et al. Intratumoral CD3 and CD8 T-cell densities associated with relapse-free survival in HCC. Cancer Immunol Res. 4(5), 419-430 (2016).

45. Fu J, Zhang Z, Zhou L et al. Impairment of CD4+ cytotoxic T cells predicts poor survival and high recurrence rates in patients with hepatocellular carcinoma. Hepatology 58(1), 139-149 (2013).

46. Xiao YS, Gao Q, Xu XN et al. Combination of intratumoral invariant natural killer $\mathrm{T}$ cells and interferon-gamma is associated with prognosis of hepatocellular carcinoma after curative resection. PLoS ONE 8(8), e70345 (2013).

47. Foerster F, Hess M, Gerhold-Ay A et al. The immune contexture of hepatocellular carcinoma predicts clinical outcome. Sci. Rep. 8(1), 5351-5351 (2018).

48. Sun L, Xu G, Liao W et al. Clinicopathologic and prognostic significance of regulatory $\mathrm{T}$ cells in patients with hepatocellular carcinoma: a meta-analysis. Oncotarget 8(24), 39658-39672 (2017).

49. Gao Q, Qiu S-J, Fan J et al. Intratumoral balance of regulatory and cytotoxic T Cells Is associated with prognosis of hepatocellular carcinoma after resection. J. Clin. Oncol. 25(18), 2586-2593 (2007).

50. Gao X-H, Tian L, Wu J et al. Circulating CD14+HLA-DR-/low myeloid-derived suppressor cells predicted early recurrence of hepatocellular carcinoma after surgery. Hepatol. Res. 47(10), 1061-1071 (2017). 
51. Arihara F, Mizukoshi E, Kitahara M et al. Increase in CD14+HLA-DR-/low myeloid-derived suppressor cells in hepatocellular carcinoma patients and its impact on prognosis. Cancer Immunol. Immunother. 62(8), 1421-1430 (2013).

52. Iwata T, Kondo Y, Kimura O et al. PD-L1(+)MDSCs are increased in HCC patients and induced by soluble factor in the tumor microenvironment. Sci. Rep. 6, 39296 (2016).

53. Zhu X-D, Zhang J-B, Zhuang P-Y et al. High expression of macrophage colony-stimulating factor in peritumoral liver tissue is associated with poor survival after curative resection of hepatocellular carcinoma. J. Clin. Oncol. 26(16), 2707-2716 (2008).

54. Capece D, Fischietti M, Verzella D et al. The inflammatory microenvironment in hepatocellular carcinoma: a pivotal role for tumor-associated macrophages. BioMed Res. Int. 2013, 15 (2013).

55. Calderaro J, Rousseau B, Amaddeo G et al. Programmed death ligand 1 expression in hepatocellular carcinoma: relationship with clinical and pathological features. Hepatology 64(6), 2038-2046 (2016).

56. Shi F, Shi M, Zeng Z et al. PD-1 and PD-L1 upregulation promotes CD8+ T-cell apoptosis and postoperative recurrence in hepatocellular carcinoma patients. Int. J. Cancer 128(4), 887-896 (2011).

57. Liu G-M, Li X-G, Zhang Y-M. Prognostic role of PD-L1 for HCC patients after potentially curative resection: a meta-analysis. Cancer Cell Int. 19, 22-22 (2019).

58. Lu C, Rong D, Zhang B et al. Current perspectives on the immunosuppressive tumor microenvironment in hepatocellular carcinoma: challenges and opportunities. Mol. Cancer 18(1), 130 (2019).

59. Zhang X, Fu X, Li T, Yan H. The prognostic value of myeloid derived suppressor cell level in hepatocellular carcinoma: a systematic review and meta-analysis. PLoS ONE 14(12), e0225327 (2019).

60. Lu LC, Chang CJ, Hsu CH. Targeting myeloid-derived suppressor cells in the treatment of hepatocellular carcinoma: current state and future perspectives. J. Hepatocell. Carcinoma 6, 71-84 (2019).

- Highlights the importance of myeloid-mediated immunosuppression in HCC.

61. McDermott DF, Huseni MA, Atkins MB et al. Clinical activity and molecular correlates of response to atezolizumab alone or in combination with bevacizumab versus sunitinib in renal cell carcinoma. Nat. Med. 24(6), 749-757 (2018).

62. Zhao HQ, Li WM, Lu ZQ, Yao YM. Roles of Tregs in development of hepatocellular carcinoma: a meta-analysis. World J. Gastroenterol. 20(24), 7971-7978 (2014).

63. Kalathil S, Lugade AA, Miller A, Iyer R, Thanavala Y. Higher frequencies of GARP ${ }^{+}$CTLA- $4^{+}$Foxp $3^{+}$T regulatory cells and myeloid-derived suppressor cells in hepatocellular carcinoma patients are associated with impaired T-cell functionality. Cancer Res. 73(8), 2435 (2013).

64. Sia D, Jiao Y, Martinez-Quetglas I et al. Identification of an immune-specific class of hepatocellular carcinoma, based on molecular features. Gastroenterology 153(3), 812-826 (2017).

-. Through analyses of gene-expression profiles, infiltrates and regulatory molecules, showed that only approximately $25 \%$ of HCCs could be classified into an 'immune class', with high levels of immune cell infiltration, expression of PD1 and/or PD-L1), activation of IFN $\gamma$ signaling, markers of cytolytic activity (such as granzyme B and perforin 1) and an absence of CTNNB1 mutations. The remainder of HCCs analysed harbored an immunosuppresive microenvironment.

65. Hilmi M, Neuzillet C, Calderaro J, Lafdil F, Pawlotsky J-M, Rousseau B. Angiogenesis and immune checkpoint inhibitors as therapies for hepatocellular carcinoma: current knowledge and future research directions. J. Immunother. Cancer 7(1), 333 (2019).

66. Li G, Staveley-O'Carroll KF, Kimchi ET. Potential of radiofrequency ablation in combination with immunotherapy in the treatment of hepatocellular carcinoma. J. Clin. Trials 6(2), 257 (2016).

67. Mizukoshi E, Yamashita T, Arai K et al. Enhancement of tumor-associated antigen-specific $\mathrm{T}$ cell responses by radiofrequency ablation of hepatocellular carcinoma. Hepatology 57(4), 1448-1457 (2013).

68. Martins-Filho SN, Paiva C, Azevedo RS, Alves VaF. Histological grading of hepatocellular carcinoma - a systematic review of literature. Front. Med. 4, 193 (2017).

69. Zhou J, Sun HC, Wang $\mathrm{Z}$ et al. Guidelines for diagnosis and treatment of primary liver cancer in China (2017 Edition). Liver Cancer 7(3), 235-260 (2018).

70. Eisenhauer EA, Therasse P, Bogaerts J et al. New response evaluation criteria in solid tumours: Revised RECIST guideline (version 1.1). Eur. J. Cancer 45(2), 228-247 (2009). 


\section{Title of article \\ IMbrave 050: A Phase III trial of atezolizumab plus bevacizumab in high-risk hepatocellular carcinoma after curative resection or ablation}

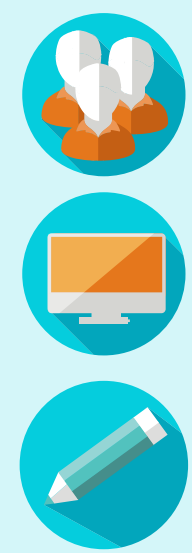

\section{Authors}

Stephen P Hack, Jessica Spahn, Minshan Chen, Ann-Li Cheng, Ahmed Kaseb, Masatoshi Kudo, Han Chu Lee, Adam Yopp, Pierce Chow \& Shukui Qin

Article URL

https://www.futuremedicine.com/doi/10.2217/fon-2020-0162

Trial registration number

NCT04102098

\section{Primary objectives/rationale}

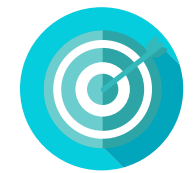

\section{Primary objective}

Demonstrate that atezolizumab combined with bevacizumab is superior to active surveillance in prolonging RFS in patients with $\mathrm{HCC}$ at high risk of recurrence following curative resection or ablation

\section{Secondary key objectives}

Evaluate overall survival, time to recurrence, RFS per investigator assessment, RFS in PD-L1-high patients, safety and tolerability and conduct correlative studies

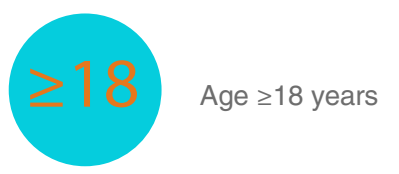

First diagnosis of HCC by radiological criteria and/or pathological confirmation, who have undergone a curative resection or ablation

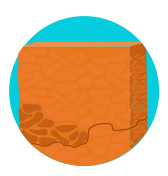

Resected HCC must have histological confirmation of negative microscopic surgical margins (R0)

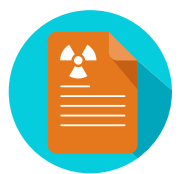

Ablated HCC must have documented response

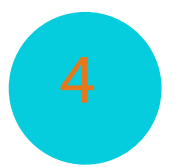

Disease-free status documented within 4 weeks prior to randomization complete radiological

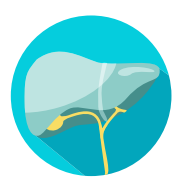

Absence of major vascular invasion of the portal vein (Vp3 or Vp4) or any grade of macrovascular invasion in the hepatic vein or inferior vena cava

No extrahepatic HCC
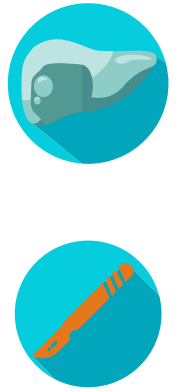

Full recovery from surgical resection or ablation within 4 weeks prior to randomization

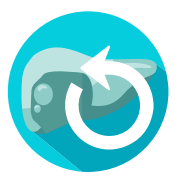

High risk for HCC recurrence

1 cycle of adjuvant TACE is permitted for resected patients if consistent with institutional guidelines

\section{Outcome measures/end points}

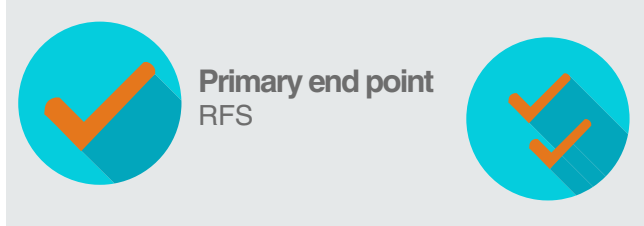

Secondary end points: OS, time to recurrence, RFS in PD-L1-high patients, safety \& tolerability and biomarkers
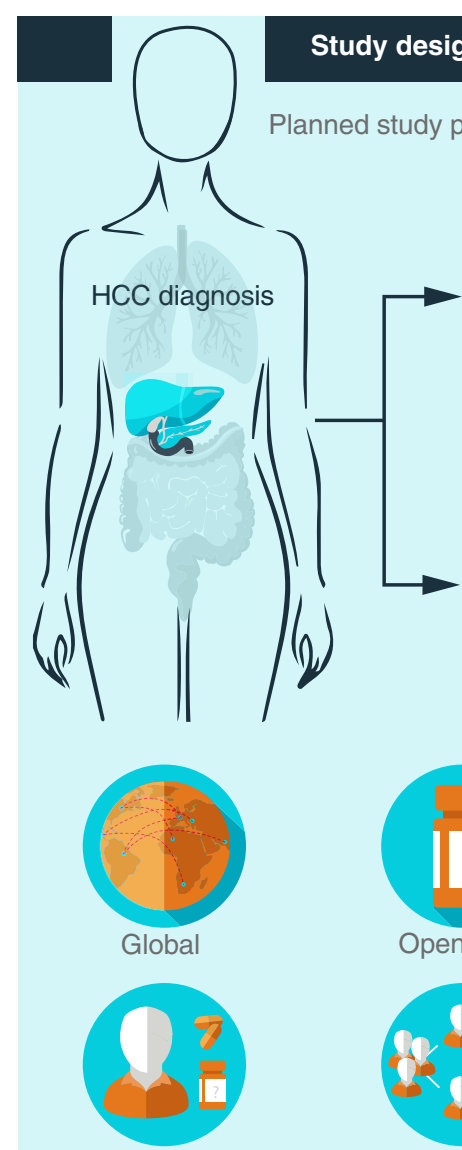

Randomized (1:1)

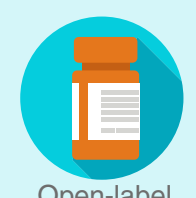

Open-label

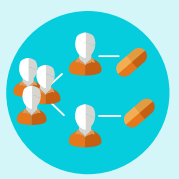

Two-arm

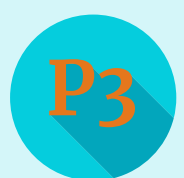

Phase III study

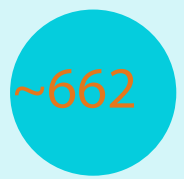

Planned sample size: approximately 662
CR: Complete response; EASL: European Association for the Study of the Liver; HCC: Hepatocellular carcinoma; IRF: Independent review facility; MWA: Microwave ablation; q3w: every 3 weeks;

R: Randomization; R0: Absence of microscopic tumor invasion of the resection margin by imaging; RFA: Radiofrequency ablation; RFS: Recurrence-free survival; OS: Overall survival; TACE: Transarterial chemoembolization 